\title{
ASSOCIAÇÃO DA HOMOCISTEÍNA E LDL EM MULHERES IDOSAS COM DIABETES DO TIPO 2
}

\author{
Alexandre de SOUZA E SILVA*1,2 \\ Amanda Heloisa RAMOS ${ }^{3}$ \\ Fábio Vieira LACERDA ${ }^{4}$; \\ Valdomiro Vagner de SOUZA $^{5}$ \\ Luís Henrique Sales OLIVEIRA ${ }^{6}$ \\ Maria Paula Gonçalves MOTA ${ }^{7}$ \\ ${ }^{1}$ Doutorando. Departamento Ciências do Desporto, Exercício e Saúde, Curso em Ciências do Desporto, Universidade \\ Trás-os-Montes Alto Douro. Vila Real - Portugal. alexprofms@yahoo.com.br \\ ${ }^{2}$ Professor de Educação Física. Mestre. Instituto de Ciências Biológicas, Curso de Educação Física, FEPI - Centro \\ Universitário de Itajubá - Itajubá (MG) - Brasil. Av. Dr. Antônio Braga Filho, 687 - Varginha - Itajubá (MG). Tel: (35) \\ 3629-8400 - E-mail: alexprofms@yahoo.com.br \\ ${ }^{3}$ Bióloga. Instituto de Ciências Biológicas, Curso de Biologia, FEPI - Centro Universitário de Itajubá. Itajubá (MG) - \\ Brasil. Tel: (35) 3629-8434 hamandahr@hotmail.com \\ ${ }^{4}$ Nutricionista e Biólogo. Mestre. Instituto de Ciências Biológicas, Curso de Educação Física, FEPI - Centro \\ Universitário de Itajubá - Itajubá (MG) - Brasil. Av. Dr. Antônio Braga Filho, 687 - Varginha - Itajubá (MG). Tel: (35) \\ 3629-8400 - E-mail: doc_fabio2004@yahoo.com.br \\ ${ }^{5}$ Farmacêutico. Mestre. Instituto de Ciências Biológicas, Curso de Farmácia, FEPI - Centro Universitário de Itajubá - \\ Itajubá (MG) - Brasil. Av. Dr. Antônio Braga Filho, 687 - Varginha - Itajubá (MG). Tel: (35) 3629-8400 - E-mail: \\ valdomirovagner@gmail.com \\ ${ }^{6}$ Fisioterapeuta. Doutor. Instituto de Ciências Biológicas, Curso de Fisioterapia, FEPI - Centro Universitário de Itajubá \\ - Itajubá (MG) - Brasil. Av. Dr. Antônio Braga Filho, 687 - Varginha - Itajubá (MG). Tel: (35) 3629-8400 - E-mail: \\ $\underline{\text { lhfisio@yahoo.com.br }}$ \\ ${ }^{7}$ Doutorada em Ciências do Desporto- Fisiologia do Exercício, Departamento Ciências do Desporto, Exercício e Saúde \\ da Universidade Trás-os-Montes Alto Douro, Centro de Investigação em Desporto, Saúde e Desenvolvimento Humano \\ (CIDESD), Vila Real - Portugal .mpmota@utad.pt
}

*Autor responsável:

Alexandre de Souza e Silva, Av. Dr. Antônio Braga Filho, 687.

Varginha - Itajubá - MG. CEP 37.501-002

Fone: 0(35) 9147 0336/3629 8400.

alexprofms@yahoo.com.br

\section{Recebido em: 10/08/2013 - Aprovado em: 1912/2013 - Disponibilizado em: 15/01/2014}

RESUMO: Diabetes do tipo 2 e as doenças cardiovasculares (DCV) são doenças com alto índice de mortalidade e morbidade. A homocisteína e a lipoproteína de baixa densidade (LDL) apresentam influência na formação das placas de ateromas. No entanto, a associação das variáveis tem sido pouco abordada na literatura, sendo importante para o controle dos riscos. O objetivo deste estudo foi avaliar a correlação entre homocisteína e LDL em mulheres idosas com diabetes do tipo 2. Para pesquisa primaria, quantitativa de corte-transversal foram recrutadas 16 mulheres idosas com diabetes do tipo 2. O grupo foi submetido à coleta de amostras sanguíneas por venopunção, sendo feitas as análises de LDL e homocisteína. Nos resultados revelam que a homocisteína não apresenta correlação com o LDL (p<0,781) e o 
IMC não apresenta relação com a homocisteína $(\mathrm{p}<0,426)$ e LDL $(\mathrm{p}<0,167)$. Foi também constatado que a pressão arterial sistólica não apresenta relação com a homocisteína $(\mathrm{p}<0,148)$ e LDL $(\mathrm{p}<0,925)$ e a pressão arterial diastólica também não apresenta associação com homocisteína $(\mathrm{p}<0,125)$ e LDL $(\mathrm{p}<0,935)$. Conclui-se que a homocisteína não apresenta associação com os níveis de LDL, IMC e pressão arterial.

Palavras-chave: idosas. diabetes. aterosclerose. homocisteína. lipoproteína de baixa densidade.

ABSTRACT: Type 2 diabetes and cardiovascular disease (CVD) are diseases with high mortality and morbidity. Homocysteine and low density lipoprotein (LDL) have an influence on the formation of atheromatous plaques. However, the association of variables and rarely addressed in the literature, it is important to control the risks. The aim of this study was to evaluate the correlation between homocysteine and LDL in older women with type 2 diabetes. For primary research, quantitative cross-section were recruited 16 elderly women with type 2 diabetes. The group underwent collection of blood samples by venipuncture, which made the analysis of LDL and homocysteine. The results found that homocysteine is not correlated with LDL $(\mathrm{P}<0.781)$ and $\mathrm{BMI}$ is not related to homocysteine $(\mathrm{P}$ $<0.426)$ and LDL $(\mathrm{P}<0.167)$. We can also observe that the systolic blood pressure is not related to homocysteine (P $<0.148)$ and LDL $(\mathrm{P}<0.925)$ and diastolic blood pressure also not associated with homocysteine $(\mathrm{P}<0.125)$ and $\mathrm{LDL}(\mathrm{P}$ $<0.935)$. It is concluded that homocysteine not associated with LDL levels, body mass index and blood pressure.

Keywords: elderly. diabetes. atherosclerosis. homocysteine. low density lipoprotein.

\section{INTRODUÇÃO}

Diabetes do tipo 2 é uma doença crônica multifatorial e afeta em média 285 milhões de pessoas. Este número deve aumentar para 439 milhões até 2030 (LAAKSON, 2011). Os pacientes com diabetes do tipo 2 têm uma probabilidade maior de desenvolver doenças cardiovasculares (LAKERVELD et al., 2008). As doenças cardiovasculares (DCV) são uma das principais causas de morte, sendo 16,7 milhões dos óbitos ocorridos em todo o mundo (MBEWU; MBANYA, 2006). A principal complicação cardiovascular que afeta os diabéticos é a aterosclerose. A aterosclerose é uma doença crônica caracterizada pelo espessamento e perda da elasticidade da parede arterial (LIBBY; THEROUX, 2005). Além dos fatores de risco já conhecidos para a formação de placas de ateroma, a homocisteína tem apresentado uma grande influência no aparecimento da doença (SEN et al., 2010; FAEH; CHIOLERO; PACCAUD, 2006). A homocisteína está associada à oxidação da lipoproteína de baixa densidade (LDL), ao comprometimento da função endotelial arterial e à formação de inflamação e trombos. Estes fatores causam complicações cardiovasculares, principalmente a aterosclerose (SEO et al., 2010). A LDL em concentrações elevadas causa disfunção endotelial que resulta na formação da placa de ateroma, sendo um fator de risco (MITRA et al., 2011). A diminuição dessa lipoproteina reduz as placas de ateroma e reduz os riscos de ter complicações secundárias decorrentes das doenças cardiovasculares (CLEARFIELD, 2010). As concentrações de homocisteína e LDL estão correlacionadas com a formação de placas de ateroma e a homocisteína pode facilitar a absorção da LDL e consequentemente a formação de placas 
nos vasos (NEVES; MACEDO; LOPES, 2004). A associação e análise dessas variáveis é pouco abordada na literatura, sendo importante para prevenção e controle dos riscos. Neste sentido, o objetivo do estudo foi analisar a correlação entre homocisteína e LDL em indivíduos com diabetes do tipo 2.

\section{METODOLOGIA}

TIPO DE PESQUISA E CARACTERIZAÇÃO DA AMOSTRA

Para pesquisa primaria, quantitativa de corte-transversal foram recrutadas 16 mulheres idosas com diabetes do tipo 2. O grupo apresentou idade entre 60 e 91 anos, com média de idade de $72,18 \pm 9,82$. Os critérios de inclusão foram pacientes com diabetes do tipo 2, idosas e que não utilizavam nenhuma droga que influenciasse nos níveis plasmáticos de homocisteína e LDL. Os critérios de exclusão foram: pacientes com infecção aguda ou crônica, com problemas hepáticos e distúrbios nutricionais.

Todos os indivíduos do estudo receberam o termo de consentimento livre e esclarecido que, após leitura, foi assinado comprovando ciência do sujeito da pesquisa de todos os riscos e benefícios a que foi exposto durante a pesquisa. O projeto $\mathrm{n}^{\circ} 139 / 9$ foi aprovado pelo Comitê de Ética e Pesquisa do Centro Universitário de Itajubá e está de acordo com a Resolução $n^{\circ}$ 196/96 do Conselho Nacional de Saúde e da Declaração de Helsinque de 1975.

\section{INSTRUMENTOS}

Para análise do LDL foi utilizado um espectrofotômetro Biospectro Benfer SP22® (leitura em $505 \mathrm{~nm}$ ), tubos e pipetas, centrífuga, cronômetro e banho-maria (MARTINS; VERÍSSIMO; SILVA, 2010; GABRIEL et al., 2004). A análise de homocisteína foi feita no equipamento de cromatografia líquida de alta performance (HPLC) que apresenta intervalo de confiança de 95\% (KOMLÓSI et al., 2010). $\mathrm{O}$ peso dos indivíduos da amostra foi verificado por uma balança da marca Filizola® (Filizola, Brasil) e para altura foi fixada na parede com parafusos um estadiômetro (marca Seca®) de PVC rígido com fita métrica metálica retrátil. $\mathrm{O}$ aparelho apresenta escala de 0 a $220 \mathrm{~cm}$ e resolução de $0,1 \mathrm{~cm}$. Para aferir a pressão arterial foi utilizado o aparelho esfigmomanômetro da marca Aneroid Sphygmomanometer-HICO HM 1001® e um estetoscópio da marca Nurse Type Professional Stethoscope-HICO HM3005® (MARTINS; VERÍSSIMO; SILVA, 2010). 


\section{PROCEDIMENTOS}

\section{ANÁLISE BIOQUÍMICA}

Inicialmente os indivíduos do estudo foram submetidos à coleta de amostras sanguíneas em jejum mínimo de 12 horas por venopunção. A veia utilizada foi antecubital, da qual foi extraído $10 \mathrm{~mL}$ de sangue. As amostras foram separadas em tubos de ensaio com EDTA (ácido etilenodiaminotetraacético) para avaliação dos níveis plasmáticos de homocisteína. Posteriormente, foram analisadas pelo método de cromatografia de alta performance (HPLC) (PFEIFFER; HUFF; GUNTER, 1999). O método enzimático por reação de ponto final foi utilizado para análise do LDL. Após a coleta, a amostra passou por triagem e foi separado o soro do plasma, sendo utilizado o soro para análise de LDL (MARTINS; VERÍSSIMO; SILVA, 2010; GABRIEL et al., 2004; ASSMANN et al., 1984).

Para aferir a pressão arterial os pacientes permaneceram em repouso de 5 minutos, sendo utilizado método auscultatório. Os indivíduos permaneceram sentados para o procedimento. Para o peso e altura os indivíduos ficaram com roupas leves e sem sapatos. O IMC foi calculado pela seguinte fórmula: peso/altura² $(\mathrm{kg} / \mathrm{m} 2)$ (MARTINS; VERÍSSIMO; SILVA, 2010).

\section{ANÁLISE ESTATÍSTICA}

$\mathrm{O}$ delineamento da pesquisa determinou a correlação nos níveis de homocisteína e LDL em indivíduos com diabetes do tipo 2. Os dados da pesquisa foram analisados quantitativamente, respectivamente por meio de técnicas estatísticas descritivas (média e desvio padrão). A média e o desvio padrão (DP) das características antropométricas dos indivíduos foram calculadas. Realizou-se o teste de correlação de Pearson, para verificar a correlação entre LDL e homocisteína. Para a correlação entre homocisteína e IMC, homocisteína e pressão arterial sistólica e diastólica, LDL e IMC e LDL e pressão arterial foi utilizado o teste de Sperman. O tratamento estatístico foi realizado no programa SPSS Statistics 20.0 e o nível de significância estabelecido para todas as situações foram a de $p=0,05$.

\section{RESULTADOS}

Caracterização da amostra é apresentada na tabela 1 (média e o desvio padrão). $\mathrm{Na}$ tabela 2, são apresentadas a correlação da homocisteína, LDL, IMC, pressão arterial sistólica e diastólica. Pode-se observar que os resultados não demonstram correlação entre as variáveis de homocisteína e LDL $(\mathrm{p}<0,781)$. O IMC não apresentou correlação entre homocisteína $(\mathrm{p}<0,426)$ e 
LDL ( $p<0,167)$ no nosso estudo. Também não foram apresentadas correlações entre a homocisteína a pressão arterial sistólica $(\mathrm{p}<0,148)$ e diastólica $(\mathrm{p}<0,125)$. Já o LDL também não apresenta relação com a pressão arterial sistólica $(\mathrm{p}<0,925)$ e diastólica $(\mathrm{p}<0,935)$

Tabela 1 - Caracterização da amostra

\begin{tabular}{lc}
\hline Variável & Média \pm DP \\
\hline Idade & $72,18 \pm 9,82$ \\
Peso & $70,68 \pm 14,38$ \\
Altura & $1,53 \pm 0,06$ \\
IMC & $30,07 \pm 5,60$ \\
& $140,62 \pm 21,7$ \\
PAS & 4 \\
PAD & $81,87 \pm 16,41$ \\
Hom & $14,88 \pm 4,96$ \\
LDL & $137,06 \pm 57,7$ \\
& 6 \\
\hline
\end{tabular}

Fonte: Próprio autor

IMC = Índice de Massa Corporal. PAS = Pressão Arterial Sistólica. PAD = Pressão Arterial Diastólica. Hom = Homocisteína. LDL = Lipoproteína de Baixa Densidade

Tabela 2 - Coeficiente de Correlação das variáveis

\begin{tabular}{|c|c|c|c|c|}
\hline \multirow{2}{*}{$\begin{array}{c}\text { Variáve } \\
1\end{array}$} & \multicolumn{2}{|c|}{ Hom } & \multicolumn{2}{|c|}{ LDL } \\
\hline & Correlaçã & $\mathrm{p}<0,0$ & Correlaçã & $\mathrm{p}<0,0$ \\
\hline & o & 5 & o & 5 \\
\hline
\end{tabular}

$\begin{array}{lllll}\text { IMC } & -0,214 & 0,426 & 0,363 & 0,167 \\ \text { PAS } & 0,379 & 0,148 & 0,026 & 0,925 \\ \text { PAD } & 0,399 & 0,125 & -0,022 & 0,935 \\ \text { Hom } & & & 0,076 & 0,781\end{array}$

LDL $\quad 0,076 \quad 0,781$

Fonte: Próprio autor

$\mathrm{p}<0,05 . \mathrm{IMC}=$ Índice de Massa Corporal. PAS $=$ Pressão Arterial Sistólica. PAD = Pressão Arterial Diastólica. Hom = Homocisteína. LDL = Lipoproteína de Baixa Densidade

\section{DISCUSSÃO}

O presente estudo analisou a correlação da homocisteína e LDL. Os resultados observados no nosso estudo demonstram que homocisteína não apresenta correlação com a LDL. Já em outros estudos foram analisados a hiperhomocisteinemia e os níveis elevados de LDL oxidada em pacientes com diabetes do tipo 2. Os resultados apresentados são diferentes do nosso estudo. Níveis elevados de homocisteína estão relacionados com os de LDL em indivíduos com diabetes do tipo 2. A homocisteína pode aumentar a rigidez arterial e facilita absorção da LDL (YUN et al., 2011; THAMPI et al., 2008; KOUBAA et al., 2007). Segundo Coppola et al. (2000), os níveis elevados de homocisteína facilitam a oxidação e a absorção das LDL devido à peroxidação lipídica gerada nas 
membranas celulares durante sua fase de auto-oxidação. As concentrações elevadas de homocisteína facilitam a absorção de LDL e consequentemente induzem a formação de placas de ateroma nos vasos (NEVES; MACEDO; LOPES, 2004). Na nossa amostra os níveis de homocisteína não eram elevados, isso pode justificar os resultados.

A homocisteína também não apresentou associação com IMC no nosso estudo. Os resultados são semelhantes aos encotrados na literatura. Níveis de homocisteína também não apresentaram relação com o IMC em mulheres pós-menopausa (YÜKSEL et al., 2011). Em outros estudos, o resultado observado foi a não correlação da homocisteína com o IMC em indivíduos com diabetes do tipo 2 (LIN et al., 2008; DE LUIS et al., 2005; SANDHU et al., 2004). O IMC também não apresentou nenhuma relação com o aumento da homocisteína em individuos com diabetes do tipo 2 com doenças cardiovasculares (OTIENO et al., 2005). O IMC, um indicado de obesidade e sobrepeso, parece não apresentar nenhuma relação com os níveis plasmáticos de homocisteína. O metabolismo da homocisteína está diretamente ligado a proteínas, e não ao metabolismo dos lipidios (NEVES; MACEDO; LOPES, 2004).

A pressão arterial sistólica e diastólica não apresentaram associação com a homocisteína. Na literatura, alguns estudos demonstram a relação da homocisteína com a pressão arterial em individuos com diabetes do tipo 2 (OTIENO et al., 2005). A homocisteína apresenta associação com a hipertensão arterial. A correlação pode ser pelo aumento da rigidez arterial e disfunção endotelial (DE LUIS et al., 2005; (VAN GULDENER; NANAYAKKARA; STEHOUWER, 2003). O aumento da homocisteína também pode estar relacionado ao aumento da pressão arterial devido a formação de placas de ateroma (KENNEDY et al., 2003). Os resultados mostram que a homocisteína está correlacionada com as pressões arteriais sistólica e diastólica (DINAVAHI et al., 2003; ARDAWI et al., 2002; DE PERGOLA et al., 2001). No nosso estudo, a diferença pode estar relacionada aos níveis mais baixos de homocisteína, o que pode não ter ocasionado alterações nos vasos.

\section{CONCLUSÃO}

O nosso estudo analisou a correlação entre homocisteína e LDL em indivíduos com diabetes do tipo 2. O resultado demonstra que não houve correlação entre homocisteína e LDL em indivíduos com diabetes do tipo 2. A homocisteína também 
não apresentou associação com o IMC e a pressão arterial no estudo. Sugerimos

\section{REFERÊNCIAS}

ARDAWI, M.S.M; ROUZI, A.A; QARI, M.H; DAHLAWI, F.M; Al-RADDADI, R.M. Influence of age, sex, folate and vitamin B12 status on plasma homocysteine in Saudis. Saudi Med J. v.23, n.8, p. 959-68, 2002. http://www.ncbi.nlm.nih.gov/pubmed/122354 71

ASSMANN G, JABS HV, KOHNERT V, NOLTE W, SCHRIEWER H. LDLcholesterol determination in blood serum following precipitation of LDL with polyvinylsulfate. Clín Chim Acta v.140, n.1, p.77-83, 1984.

http://www.ncbi.nlm.nih.gov/pubmed/674462 9

CLEARFIELD, M.B. Altering the pathophysiology of atherosclerosis: the multidimensional role of statins. J Am Osteopath Assoc. v.110, n.4, p.2-6, 2010. http://www.ncbi.nlm.nih.gov/pubmed/204531 92

COPPOLA, A; DAVI, G; DE STEFANO, V; MANCINI, F.P; CERBONE, A.M; DI MINNO, G. Homocysteine, coagulation, platelet function, and thrombosis. Semin Thromb Hemost. v.26, n.3, p.243-254, 2000. doi: $10.1055 / \mathrm{s}-2000-8469$.

http://www.ncbi.nlm.nih.gov/pubmed/110118 42

DE LUIS, D.A; FERNANDEZ, N; ARRANZ, M.L; ALLER, R; IZAOLA, O; ROMERO, E. Total homocysteine levels relation with chronic complications of diabetes, body composition, and other estudos que incluam indivíduos com níveis elevados de homocisteína. cardiovascular risk factors in a population of patients with diabetes mellitus type 2 . J

Diabetes Complications. v.19, n.1, p. 42-6, 2005. doi: 10.1016/j.jdiacomp.2003.12.003. http://www.ncbi.nlm.nih.gov/pubmed/156424 89

DE PERGOLA, G; PANNACCIULLI, N; ZAMBONI, M; MINENNA, A; BROCCO, G; SCIARAFFIA, M; BOSELLO, G.R. Homocysteine plasma levels are independently associated with insulin resistance in normal weight, overweight and obese pre-menopausal women. Diabetes Nutr Metab. v.14, n.5, p. 253-258, 2001. http://www.ncbi.nlm.nih.gov/pubmed/118064 65

DINAVAHI, R; COSSROW, N; KUSHNER, H; FALKNER, B. Plasma homocysteine concentration and blood pressure in young adult African Americans. Am J Hypertens. v.16,n.9,p. 767-770, 2003. http://www.ncbi.nlm.nih.gov/pubmed/129440 36

FAEH, D; CHIOLERO, A; PACCAUD, F. Homocysteine as a risk factor for cardiovascular disease: should we (still) worry about? Swiss Med Wkly. v.136,n.4748,p.745-756, 2006. doi: 2006/47/smw11283. http://www.ncbi.nlm.nih.gov/pubmed/172251 94

GABRIEL, R; SAIZ, C; SUSI, R; ALONSO, M; VEGA, S; LÓPEZ, I; CRUZ CARDENAL, M.M; GÓMEZ-GERIQUE, J.Á; PORRES, A; MUÑIZ, J. Epidemiology of lipid profile of the Spanish elderly 
population: the EPICARDIAN study. Med Clin. v.122, n.16, p.605-609, 2004. http://www.ncbi.nlm.nih.gov/pubmed/151425 07

KENNEDY, B.P; FARAG, N.H; ZIEGLER, M.G; MILLS, P.J. Relationship of systolic blood pressure with plasma homocysteine: importance of smoking status. J Hypertens. v.21, n.7, p. 1307-1312, 2003.

http://www.ncbi.nlm.nih.gov/pubmed/128171 77

KOMLÓSI, V; HITRE, E; PAP, E; ADLEFF, V; RÉTI, A; SZÉKELY, E; BIRÓ, A; RUDNAI, P; SCHOKET, B; MÜLLER, J; TÓTH, B; OTTÓ, S; KÁSLER, M; KRALOVÁNSZKY, J; BUDAI, B. SHMT1 1420 and MTHFR 677 variants are associated with rectal but not colon cancer. BMC

Cancer. p.10. 2010.doi: 10.1186/1471-240710-525

http://www.ncbi.nlm.nih.gov/pubmed/209203 50

KOUBAA, N; NAKBI, A; SMAOUI, M; ABID, N; CHAABA, R; ABID, M, MAMMAMI, M. Hyperhomocysteinemia and elevated ox-LDL in Tunisian type 2 diabetic patients: role of genetic and dietary factors.

Avicene,Clin Biochem. v.40, n.13-14, p.1007-1014, 2007.http://www.ncbi.nlm.nih.gov/pubmed/1 7618615

LAAKSO, M. Heart in diabetes: a microvascular disease. Finland, Kuopio: Department of Medicine, University of Eastern Finland, Diabetes care, v. 34, n.2, p. 145-49, 2011. doi: 10.2337/dc11-s209. http://www.ncbi.nlm.nih.gov/pubmed/215254 46

LAKERVELD, J; BOT, S.D; CHINAPAW, M.J; VAN TULDER, M.W; VAN OPPEN, P; DEKKER, J.M; NIJPELS, G.Primary prevention of diabetes mellitus type 2 and cardiovascular diseases using a cognitive behavior program aimed at lifestyle changes in people at risk: Design of a randomized controlled trial. BMC Endocrine Disorders. v.8, n. 6, 2008. doi: 10.1186/1472-6823-8-6. http://www.ncbi.nlm.nih.gov/pubmed/185732 21

LIBBY, P; THEROUX, P. Pathophysiology of coronary artery disease. Circulation. $p$. 3481-3488, 2005.

LIN, Y.H; PAO, K.Y; YANG, W.S; WU, V.C; CHEN, Y.J; LIN, Y.L; TSAI, W.S; TSAI, I.J; GAU, C.S; HWANG, J.J. Waist-tohip ratio correlates with homocysteine levels in male patients with coronary artery disease. Clin Chem Lab Med. v.46, n.1, p.125-130, 2008. doi: 10.1515/CCLM.2008.016. http://www.ncbi.nlm.nih.gov/pubmed/181940 83

LYRA, R; OLIVEIRA, M; LINS, D; CAVALCANTI, N. Prevention of type 2 diabetes mellitus. Pernambuco, Recife: Faculdade de Ciências Médicas de Pernambuco, Universidade de Pernambuco, Arq Bras Endocrinol Metab. v.50, n.2, p. 239-249, 2006. http://dx.doi.org/10.1590/S000427302006000200010. http://www.scielo.br/scielo.php?pid=S000427302006000200010\&script=sci_arttext\&tlng $=\mathrm{pt}$

MARTINS, R. A; VERÍSSIMO, M. T; SILVA, M. J. C; CUMMING, S.P; TEIXEIRA, A.M. Effects of aerobic and strength-based training on metabolic health indicators in older adults. Lipids Health Dis. v.9, n.76, p.1-6, 2010. doi: 10.1186/1476511X-9-76.

http://www.ncbi.nlm.nih.gov/pmc/articles/PM C2912308/

MBEWU, A; MBANYA, J.C.

Cardiovascular Disease. $2^{\mathrm{a}}$ ed. Washington, DC: World Bank, 2006.

http://www.ncbi.nlm.nih.gov/pubmed/212906 55

MITRA, S; DESHMUKH, A; SACHDEVA, R; LU, J; MEHTA, J.L. Oxidized low-density 
lipoprotein and atherosclerosis implications in antioxidant therapy. Am J Med Sci. v.342, n.2, p.135-142, Aug. 2011. doi: 10.1097/MAJ.0b013e318224a147. http://www.ncbi.nlm.nih.gov/pubmed/217472 78

NEVES, L.B; MACEDO, D.M; LOPES, A.C. Homocisteína. J Bras Patol Med Lab. v.40, n.5, p.311-320, 2004.

OTIENO, C.F; VAGHELA, V; OGOLA, E.N; AMAYO, E.O. Patterns of homocysteine in Kenyans with type 2 diabetes without overt cardiovascular disease at Kenyatta National Hospital, Nairobi. Kenya, Nairobi. East Afr Med J. v.82, n.12, p.180-183, 2005.

http://www.ncbi.nlm.nih.gov/pubmed/166197 30

PFEIFFER, C.M; HUFF, D.L; GUNTER, E.W. Rapid and accurate HPLC assay for plasma total homocysteine and cysteine in a clinical laboratory setting. Clin Chem. v.45, n.2, p.290-292, 1999.

http://www.ncbi.nlm.nih.gov/pubmed/993105 6

SANDHU, J.S; SINGH, L; AGGARWAL, S.P; NARANG, A.P; SANDHU, P. Plasma homocysteine and insulin in diabetic nephropathy: relationship to body mass index.

Ren Fail. v.26, n.6, p. 689-693, 2004. http://www.ncbi.nlm.nih.gov/pubmed/156002 61

SEN, S; REDDY, P.L; GREWAL, R.P; BUSBY, M; CHANG, P; HINDERLITER, A. Hyperhomocysteinemia is Associated with Aortic Atheroma Progression in Stroke/TIA Patients. Front Neurol. v.1, p. 131, 2010. doi: 10.3389/fneur.2010.00131.

http://www.ncbi.nlm.nih.gov/pmc/articles/PM C3008913/

SEO, H; OH, H; PARK, H, PARK, M; JANG, Y; LEE, M. Contribution of dietary intakes of antioxidants to homocysteineinduced low density lipoprotein (LDL) oxidation in atherosclerotic patients. Yonsei Med J. v.51, n.4, p.526-533, 2010. doi: 10.3349/ymj.2010.51.4.526.

http://www.ncbi.nlm.nih.gov/pubmed/204994 17

THAMPI, P; STEWART, B.W; JOSEPH, L; MELNYK, S.B; HENNINGS, L.J; NAGARAJAN, S. Dietary homocysteine promotes atherosclerosis in apoE-deficient mice by inducing scavenger receptors expression. Atherosclerosis. v.197, n.2, p.620-629, 2008.

http://www.ncbi.nlm.nih.gov/pubmed/179502 95

VAN GULDENER, C; NANAYAKKARA, P.W; STEHOUWER, C.D. Homocysteine and blood pressure. Curr Hypertens Rep. v.5, n.1, p. 26-31, 2003.

http://www.ncbi.nlm.nih.gov/pubmed/125309 32

YÜKSEL, H. COBANOGLU, M; ODABASI, A.R; SEZER, S.D; KÜÇÜK, M; SERTER, M. Effects of early initiated oestrogen replacement therapy on plasma homocysteine levels in women in surgically induced menopause. Gynecol Endocrinol. v.27, n.2, p.101-106, 2011. doi:10.3109/09513590.2010.487610. http://www.ncbi.nlm.nih.gov/pubmed/205040 97

YUN, J; KIM, J.Y; KIM, O.Y; JANG, Y; CHAE, J.S; KWAK, J.H; LIM, H.H; PARK, H.Y; LEE, S.H; LEE, J.H. Associations of plasma homocysteine level with brachialankle pulse wave velocity, LDL atherogenicity, and inflammation profile in healthy men. Nutrition, Metabolism \& Cardiovascular Diseases. p. 136-143, Feb. 2011. doi: 10.1016/j.numecd.2009.08.003. http://www.ncbi.nlm.nih.gov/pubmed/198540 35 